\title{
Phase Behavior of Polyelectrolyte-Surfactant Complexes at Planar Surfaces
}

\author{
Adi Shafin $*$ and David Andelmant \\ School of Physics and Astronomy \\ Raymond and Beverly Sackler Faculty of Exact Sciences \\ Tel Aviv University, Ramat Aviv, Tel Aviv 69978, Israel
}

(Dated: 07 August 2006)

\begin{abstract}
We investigate theoretically the phase diagram of an insoluble charged surfactant monolayer in contact with a semi-dilute polyelectrolyte solution (of opposite charge). The polyelectrolytes are assumed to have long-range and attractive (electrostatic) interaction with the surfactant molecules. In addition, we introduce a short-range (chemical) interaction which is either attractive or repulsive. The surfactant monolayer can have a lateral phase separation between dilute and condensed phases. Three different regimes of the coupled system are investigated depending on system parameters. A regime where the polyelectrolyte is depleted due to short range repulsion from the surface, and two adsorption regimes, one being dominated by electrostatics, whereas the other by short range chemical attraction (similar to neutral polymers). When the polyelectrolyte is more attracted (or at least less repelled) by the surfactant molecules as compared with the bare water/air interface, it will shift upwards the surfactant critical temperature. For repulsive short-range interactions the effect is opposite. Finally, the addition of salt to the solution is found to increase the critical temperature for attractive surfaces, but does not show any significant effect for repulsive surfaces.
\end{abstract}

PACS numbers: 82.35.Gh, 82.35.Rs, 61.41.+e

\section{INTRODUCTION}

Complexation between surfactants and macromolecules has generated a great deal of research, both theoretically 1, 2, 3, 4, 5, 6, 7, 8, 9, 10, 11, 12, 13, 14] and experimentally [15, 16, 17, 18, 19, 20, 21, 22, 23]. This research is motivated in part by the role surfactantmacromolecule complexes plays in biological systems 8, 10], where the adsorption of a variety of macromolecules onto charged lipid cell membranes is a precursor to many important biological processes.

The complexation of charged surfactants and macromolecules, besides its relevance to biological physics, carries interesting questions about the interplay of short-range interactions between surfactants and macromolecules, as compared with long-range electrostatic interactions. We consider a multicomponent monolayer where the possibility of a lateral phase demixing between the various constitutes exists and is characterized by a demixing (miscibility) curve in the temperatureconcentration plane. For very long polyelectrolyte (PE) chains, the electrostatic interactions have two opposite effects on the adsorption. Electrostatic attraction between surfactants and PE chains increases the complexation, while the electrostatic repulsion between the PE monomers tends to decrease the complexation. This interplay also stimulated the interest in the adsorption of polyelectrolytes on oppositely charged surfaces [24, 25, 26, 27, 28, 29, 30, 31, 32, 33, 34, 35, 36, 37].

Several authors have addressed a closely related prob-

*Electronic address: shafira@post.tau.ac.il

${ }^{\dagger}$ Electronic address: andelman@post.tau.ac.il lem of neutral polymers adsorbing on a surfactant monolayer $3,8,10]$. It was shown that for polymer-surfactant systems the surfactant demixing occurs for higher temperatures as compared to a pure surfactant system. Other works dealt with another similar problem of globular proteins (rigid macromolecules with no flexibility) adsorbed on charged membranes 4, 5]. A recent paper 9] also deals with the adsorption of one flexible polyelectrolyte chain on a surfactant monolayer using Monte Carlo simulations. The possibility that the polyelectrolytes can desorb entirely from the charged surface when the solution has sufficiently strong ionic strength [33] was not previously discussed.

In this paper, we analyze the demixing of surfactants on the monolayer plane using numerical solutions of the mean-field equations. We find that the short-range interactions between the PE and the surfactants have a greater effect on the shape of the phase separation curve than the long-range electrostatics. Three regimes for the surfactant-polyelectrolyte complexes are found: (i) a depletion regime, where the polyelectrolytes do not adsorb at all onto the surfactant layer; (ii) an electrostatic adsorption regime, where the main attractive interaction between surfactants and polyelectrolytes is the electrostatic attraction; and (iii) a chemical adsorption regime, where the main interaction between the polyelectrolytes and the surface is short-ranged (chemical) and attractive. In the first two regimes, the surfactant demixing curve (binodal line) decreases in comparison to that of the pure surfactant monolayer, while in the third regime the demixing curve increases. We also find that for the electrostatic adsorption regime, the adsorbed amount for a system in the coexistence region is almost the same as a monophasic system with the same average concentration. In the chemical adsorption case the adsorbed amount in- 
creases more substantially due to the phase demixing.

The outline of this paper is as follows: we start by introducing the free-energy functional for a PE solution with salt in contact with a charged surfactant monolayer. In Sec. IIII we present our numerical results and discussion. In Sec. IV we present a simple scaling analysis for three limits: (i) PE depletion from a short-range repulsive surface; (ii) PE electrostatic adsorption to a shortrange repulsive surface; and (iii) the short-range attractive surface case. Finally, we present some concluding remarks in Sec. D

\section{FREE ENERGY FORMULATION}

Consider an aqueous solution of polyelectrolyte chains and salt ions. The bulk PE solution is in contact with a planar and rigid interface, on which resides an insoluble monolayer of charged surfactants. This monolayer can be thought of as a Langmuir monolayer at the air-water interface, or as the outer leaflet of a lipid bilayer membrane. The charged surfactants are assumed to be able to move freely on the interface, but can not dissolve into the solution (see Figure 1).

The free energy of such a system is composed of three contributions. The monolayer free energy $F_{\text {surf }}$, the solution free energy $F_{\text {sol }}$ and the interaction free energy $F_{\text {int }}$. We use a standard lattice-gas formalism [2] to derive the demixing free energy of the surfactant monolayer:

$$
\begin{array}{r}
\frac{F_{\text {surf }}}{k_{B} T}=b^{-2}[c \ln c+(1-c) \ln (1-c) \\
\left.+\nu^{-1} c(1-c)-c Z \zeta_{s}\right],
\end{array}
$$

where $b^{2}$ is the close packing area of the surfactant head groups. The first two terms account for the surfactant entropy of mixing, where $c$ is the surfactant area fraction. The third term represents a short-range interaction between the surfactants with $\nu^{-1}$ as the interaction parameter. The fourth term is electrostatic contribution of the charged surfactant layer, where $Z$ is the valency of the surfactant head group (assumed henceforth to be negative) and $\zeta_{s}=e \psi(0) / k_{B} T$ is the surface potential on the monolayer plane in units of $k_{B} T$.

Without the fourth term, $F_{\text {surf }}$ is the free energy commonly used for neutral Langmuir monolayers. For simplicity, we take it to be symmetric under the exchange of $c$ and $1-c$. The non-convexity of $F_{\text {surf }}$ implies that there is a demixing region with two phases: (i) a dense phase with high surfactant concentration, and (ii) a dilute phase with low surfactant concentration. The phase demixing curve is determined by:

$$
\nu_{b}=\frac{1-2 c}{\ln (1-c)-\ln c},
$$

where $\nu=\nu_{b}(c)$ is the binodal line. Below a critical value $\nu_{c}=1 / 2$ and for $\nu(c)<\nu_{b}(c)$ the monolayer phase sep-

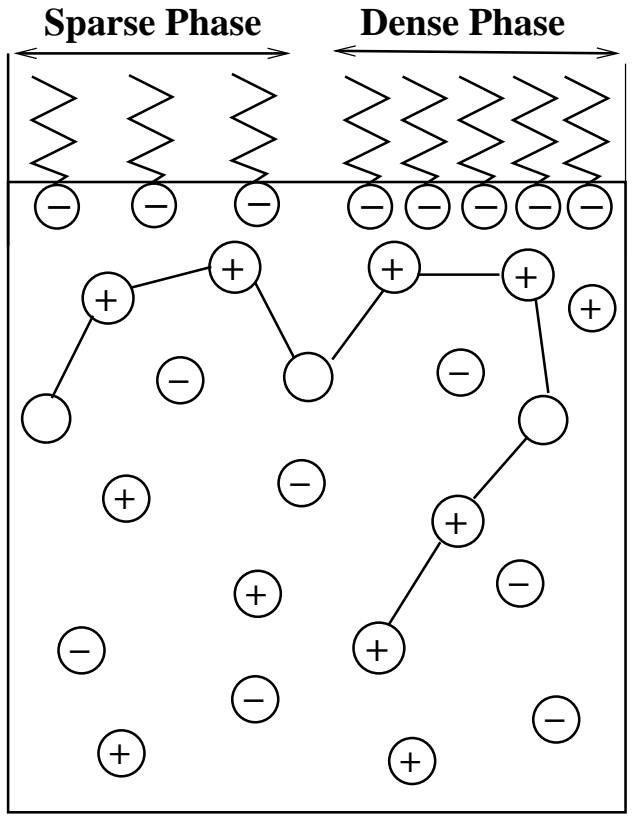

FIG. 1: A schematic view of the polyelectrolyte-surfactant system. The polyelectrolyte solution contains polyelectrolyte chains and monovalent salt (co- and counterions). The negatively charged surfactants are present only on the air-water interface, and phase separate into dense and dilute lateral phases.

arates into two coexisting phases. Inside the coexistence region, another line of interest is the spinodal line, satisfying $F_{\text {surf }}^{\prime \prime}(c)=0$. The spinodal line, separating the unstable and metastable regions with respect to surfactant density fluctuations, is given by:

$$
\nu_{\mathrm{sp}}=2 c(1-c) .
$$

Its maximum coincides with that of the binodal line at the critical point, $c_{c}=0.5$ and $\nu_{c}=0.5$. Therefore, examination of the spinodal line gives a good initial approximation to the phase behavior.

The free energy of the bulk $\mathrm{PE}$ and salt solution, $F_{\text {sol }}=$ $F_{\text {pol }}+F_{\text {ions }}+F_{\text {el }}$, is more complex, and is composed of three parts: (i) the polymer free energy $F_{\text {pol }}$; (ii) the small ion entropy $F_{\text {ions }}$; and (iii) the electrostatic free energy $F_{\text {el }}$. The polymer free energy is [30]:

$$
\frac{F_{\mathrm{pol}}}{k_{B} T}=\int_{0}^{\infty} \mathrm{d} x\left[\frac{a^{2} \phi_{b}^{2}}{6}\left|\frac{\mathrm{d} \eta}{\mathrm{d} x}\right|^{2}+\frac{1}{2} v_{\mathrm{ex}} \phi_{b}^{4}\left(\eta^{2}-1\right)^{2}\right],
$$

where the first term accounts for the polymer chain flexibility with $a$ the monomer size, $\phi_{b}^{2}$ the bulk concentration of monomers and $\eta^{2}(x) \equiv \phi^{2} / \phi_{b}^{2}$ the local monomer concentration renormalized by its bulk value. The parameter $v_{\mathrm{ex}}$ is the polymer excluded volume (second virial) coefficient. The second term originates from the excluded volume interactions between the monomers as well as the 
equilibrium condition of the PE solution in contact with a bulk reservoir.

The change in small ion entropy with respect to the reservoir is:

$$
\frac{F_{\text {ions }}}{k_{B} T}=\sum_{i= \pm} \int_{0}^{\infty} \mathrm{d} x\left[c_{i} \ln \frac{c_{i}(x)}{c_{b}^{i}}-c_{i}(x)+c_{b}^{i}\right]
$$

where $c_{ \pm}(x)$ is the local concentration of the positive and negative ions and $c_{b}^{ \pm}$is their bulk concentration.

The last term of $F_{\text {sol }}$, the electrostatic energy, is:

$$
\begin{aligned}
& \frac{F_{\mathrm{el}}}{k_{B} T}= \\
& \quad \int_{0}^{\infty} \mathrm{d} x\left[\left(c_{+}-c_{-}+f \phi_{b}^{2} \eta^{2}\right) \zeta-\frac{1}{8 \pi l_{B}}\left(\frac{\mathrm{d} \zeta}{\mathrm{d} x}\right)^{2}\right]
\end{aligned}
$$

where the dimensionless electrostatic potential (rescaled by the temperature) is $\zeta \equiv e \psi / k_{B} T$. The first three terms are the interaction of the electrostatic potential with the positive ion, negative ion and polyelectrolyte concentrations, respectively, where the parameter $f$ is the fraction of charged monomers on the PE chain. The last term is the self-energy of the electric field, where $l_{B}=$ $e^{2} / \varepsilon k_{B} T$ is the Bjerrum length. For water with dielectric constant $\varepsilon=80$ at room temperature $T=300 \mathrm{~K}, l_{B}$ is equal to about $7 \AA$.

The last contribution to the free energy, the nonelectrostatic interaction free energy $F_{\text {int }}$, can be written as [3] :

$$
\frac{F_{\text {int }}}{k_{B} T}=-\frac{1}{2} b^{-2} \varepsilon_{\mathrm{ps}}\left(c-c^{*}\right) \phi_{b}^{2} \eta_{s}^{2}
$$

In the above $\eta_{s} \equiv \eta(0)$ is the renormalized monomer concentration at the surface, $x=0$. The strength of the PEsurfactant interaction is defined by a phenomenological parameter $\varepsilon_{\mathrm{ps}}$, which has usints of volume. For positive values of $\varepsilon_{\mathrm{ps}}$, the short-range interaction of the PEs with the surfactant is more favorable than those between the $\mathrm{PE}$ and the bare interface, while for $\varepsilon_{\mathrm{ps}}<0$ the opposite occurs. At concentration $c=c^{*}$ the surface is indifferent ("special transition" line) [1, 3]. For $\varepsilon_{\mathrm{ps}}>0$ and low surfactant concentrations $c<c^{*}$, the short-range interactions between the surface and the PE are repulsive, while for higher surfactant concentrations $c>c^{*}$ these interactions are attractive.

In order to find the equilibrium state of the PEsurfactant system we minimize the total free energy, $F=F_{\text {surf }}+F_{\text {pol }}+F_{\text {ions }}+F_{\text {el }}+F_{\text {int }}$ with respect to the local small ion densities $c_{ \pm}$, electrostatic potential $\zeta$ and monomer order parameter $\eta$. This minimization yields the following equations $30,31,33$ :

$$
\frac{\mathrm{d}^{2} \zeta}{\mathrm{d} x^{2}}=\kappa^{2} \sinh \zeta+k_{m}^{2}\left(\mathrm{e}^{\zeta}-\eta^{2}\right)+\frac{4 \pi l_{B} c Z}{b^{2}} \delta(x)
$$

$$
\frac{a^{2}}{6} \frac{\mathrm{d}^{2} \eta}{\mathrm{d} x^{2}}=v \phi_{b}^{2}\left(\eta^{3}-\eta\right)+f \zeta \eta-\frac{\varepsilon_{\mathrm{ps}}\left(c-c^{*}\right)}{2 b^{2}} \eta \delta(x),
$$

where $\kappa^{-1}=\left(8 \pi l_{B} c_{\text {salt }}\right)^{-1 / 2}$ is the Debye-Hückel length scale for the screening of the electrostatic potential in the presence of added salt and $k_{m}^{-1}=\left(4 \pi l_{B} \phi_{b}^{2} f\right)^{-1 / 2}$ is the corresponding length for the potential decay due to counterions. Note that the actual decay of the electrostatic potential is determined by a combination of salt, counterions, and polymer screening effects.

The solution of Eqs. (8) and (9) requires four boundary conditions. Two of them are chosen at the bulk, far from the surfactant monolayer, and two at the surfactant layer. At the bulk we set the electrostatic potential as $\zeta(x \rightarrow \infty)=0$, while the monomer concentration is set equal to the bulk monomer concentration $\eta(x \rightarrow \infty)=1$. The other two boundary conditions are obtained by integrating Eqs. (8) and (9) from 0 to a small distance near the surface incorporating the surface interactions of Eqs. (8), (9):

$$
\begin{aligned}
& \left.\frac{\mathrm{d} \zeta}{\mathrm{d} x}\right|_{x=0}=\frac{4 \pi l_{B} c Z}{b^{2}} \\
& \left.\frac{\mathrm{d} \eta}{\mathrm{d} x}\right|_{x=0}=-\frac{3 \varepsilon_{\mathrm{ps}}\left(c-c^{*}\right)}{b^{2} a^{2}} \eta(0)
\end{aligned}
$$

Equation (10) is the usual electrostatic boundary condition for a given surface charge density $|\sigma| \equiv c Z / b^{2}$, while Eq. (111) is the Cahn - de Gennes boundary condition [38], which is often used for neutral polymers 29].

Equations (8)-(11) are solved numerically using the relaxation method 39], as was described previously 33]. For each surfactant concentration, we calculate the value of $\nu$ for which $F^{\prime \prime}(c)=0$, corresponding to the spinodal curve. Then the full binodal line is calculated numerically. We find that despite the electrostatic interactions between the surfactants and the PE chains, the main characteristics of the spinodal and binodal lines depend on the short-range (non-electrostatic) PE-surfactant interactions. There are two main regimes for the demixing curve. For large and positive $\varepsilon_{\mathrm{ps}}\left(c-c^{*}\right)$ values (shortrange attractive surface), the demixing curve is shown to be always higher than the pure monolayer one, Eq. (3). For large negative $\varepsilon_{\mathrm{ps}}\left(c-c^{*}\right)$ values (short-range repulsive surface), the curve is always lower than the pure one, Eq. (3).

In order to incorporate the temperature dependence of the various parts, we note that $\nu$, the inverse FloryHuggins parameter, is proportional to the temperature $T$. The chemical interaction strength parameter $\varepsilon_{\mathrm{ps}}$ is proportional to $T^{-1}$. The other parts of the free energy have a weaker dependence on $T$, which can be ignored in this simple model 3]. Their contribution is neither purely enthalpic nor purely entropic, and is less significant. Therefore, in order to simulate the change in temperature in our simple model, we follow the method shown previously in Ref. [3] and choose $\nu \cdot \varepsilon_{\mathrm{ps}}=\Theta$ to be constant. 


\section{NUMERICAL RESULTS}

\section{A. The Three PE-Surfactant Regimes}

We find three distinct regimes for the PE-surfactant system. These regimes are presented in Fig 2, and discussed more thoroughly in Sec. [IV] For repulsive surfaces, very low $c$ and high $c_{\text {salt }}$, the PEs deplete from the interface. The high amount of salt screens the electrostatic interactions between the constituents of the solution, and the free energy can be written as a sum of three terms: an interface term, a neutral polymer term and a salt solution term. For higher $c$, short-range repulsive surfaces and low salt, the PEs adsorb electrostatically on the surface. In this case, the free energy is dominated by the electrostatic attraction between the PE chains and the surfactant layer. For the opposite case of sort-range attractive surfaces, the adsorption is dominated by the short-range attraction.

In Fig. 2a we present the phase diagram in the plane $\left(c_{\text {salt }}, c\right)$ differentiating the three regimes in the case of $\varepsilon_{\mathrm{ps}}>0$, namely, when the surfactant-PE interaction is more favorable than the PE - bare surface interaction. For $c<c^{*}$, in the area marked E, the short-range interaction between the surface and the PE chains is repulsive, and the adsorption is dominated by the electrostatic attraction between the PEs and the surfactants. For high amounts of added salt, this electrostatic attraction is screened, and the PE chains deplete (marked D). For $c>c^{*}$, (marked C) the PE chains chemically adsorb on the surface for all $c_{\text {salt }}$. The electrostatic interactions in this region are dominated by the monomer-monomer electrostatic repulsion, and hence the addition of salt increases the adsorption, rather than causing depletion. In Fig. $2 \mathrm{~b}$ we show the opposite case of $\varepsilon_{\mathrm{ps}}<0$. In this case the line separating the phases is no longer monotonic. For $c<c^{*}$, the adsorption is mainly chemical, and is enhanced by addition of salt. For $c>c^{*}$, the adsorption becomes electrostatic and depends on $c_{\text {salt }}$. The amount of salt necessary for depletion decreases with $c$ for low $c$ since the increase in $c$ increases the short-range repulsion between the surface and the PE chains, while the surface charge is not strong enough to electrostatically attract the PEs. For higher $c$, the increase in the surface charge with $c$ increases mainly the electrostatic adsorption, and the necessary amount of salt increases with $c$.

\section{B. The Demixing Curve}

Repulsive Surface The spinodal lines of repulsive PEsurfaces $\left(c^{*}=3.0, \nu \cdot \varepsilon_{\mathrm{ps}}=0.5 b^{2} a\right)$ for several values of $c_{\text {salt }}$ are presented in Fig. 3a. The spinodal of the pure surface monolayer, Eq. (3), is shown to be always higher than any of the charged-surface spinodals. All three spinodal lines are almost symmetric under the change $c \rightarrow 1-c$, and the critical points are very close to the
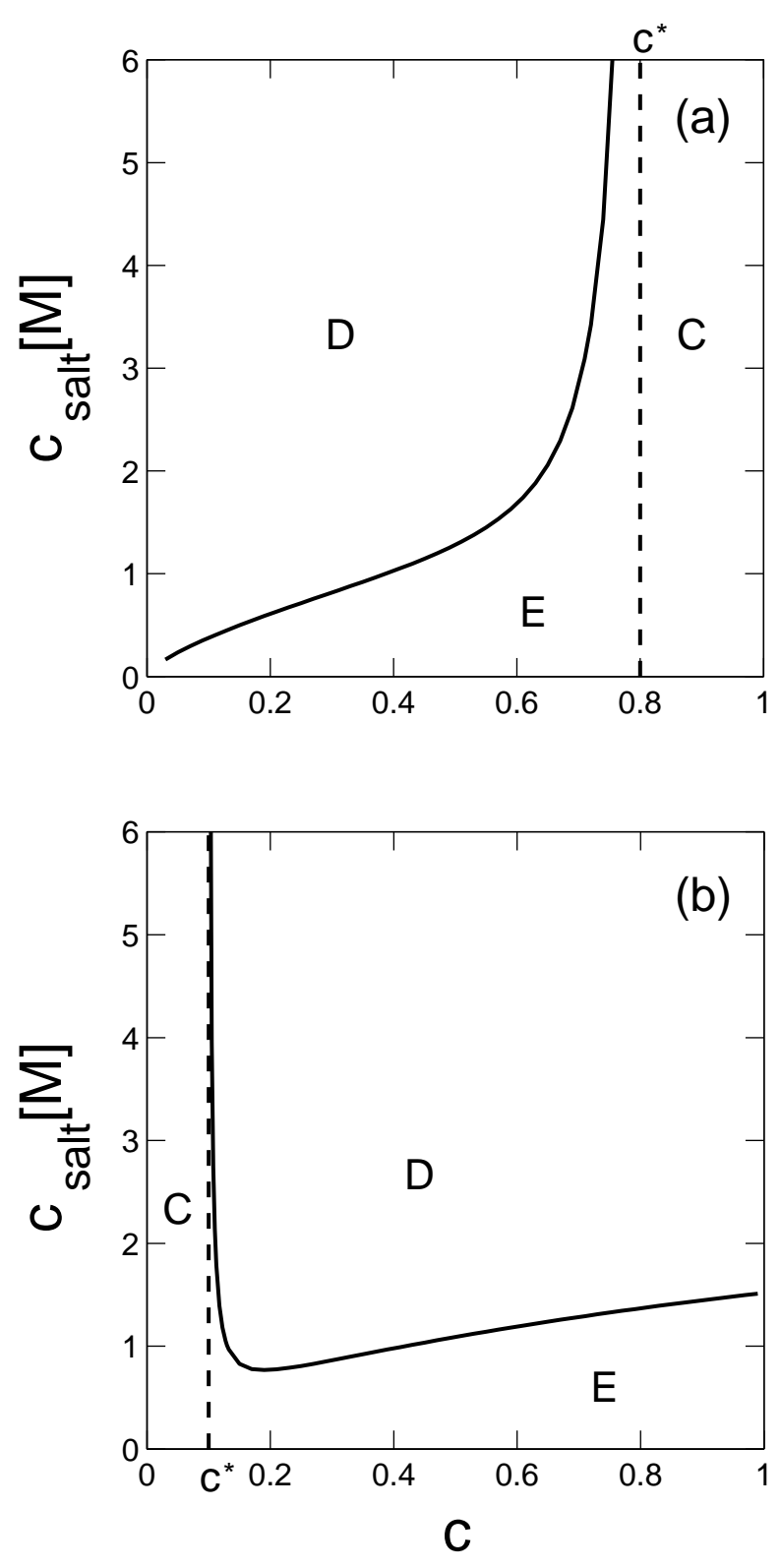

FIG. 2: The numerically calculated phase diagram of the three PEsurfacant regimes are presented for two values of $\nu \cdot \varepsilon_{\mathrm{ps}}$ and $c^{*}$. In both figure parts the solid line differentiates the electrostatic adsorption regime (marked E) from the depletion regime (D), and the dashed line differentiates both of them from the chemical adsorption regime (marked C). In (a) we set $\nu \cdot \varepsilon_{\mathrm{ps}}=0.5 b^{2} a$ and $c^{*}=0.8$, meaning that the short-range interaction of the PE chains with the charged surfactants is more favorable. For $c<c^{*}$ and low salt concentrations, the electrostatic attraction between the surfactants and the PE chains causes them to adsorb on the monolayer. For higher salt concentrations the PE and surface charges are screened by the salt ions, and the PE chains deplete. For $c>c^{*}$, the short-range attraction between the surfactants and the PE chains is strong enough to allow PE adsorption for any salt concentration. In (b) we show the opposite case of $\nu \cdot \varepsilon_{\mathrm{ps}}=-0.9 b^{2} a$ and $c^{*}=0.1$, where the short-range interactions with the surfactants are less favorable. Here for $c<c^{*}$ the shortrange interactions cause the $\mathrm{PE}$ to adsorb, while for $c>c^{*}$ the $\mathrm{PE}$ chains adsorb or deplete according to the strength of the electrostatic interactions and the added salt concentration. In both plots we use
$\nu=0.1, a=5 \AA \quad v_{\text {ex }}=10 \AA^{3}, \phi^{2}=10^{-8} \AA^{-3} Z=1, f=0.5$. 

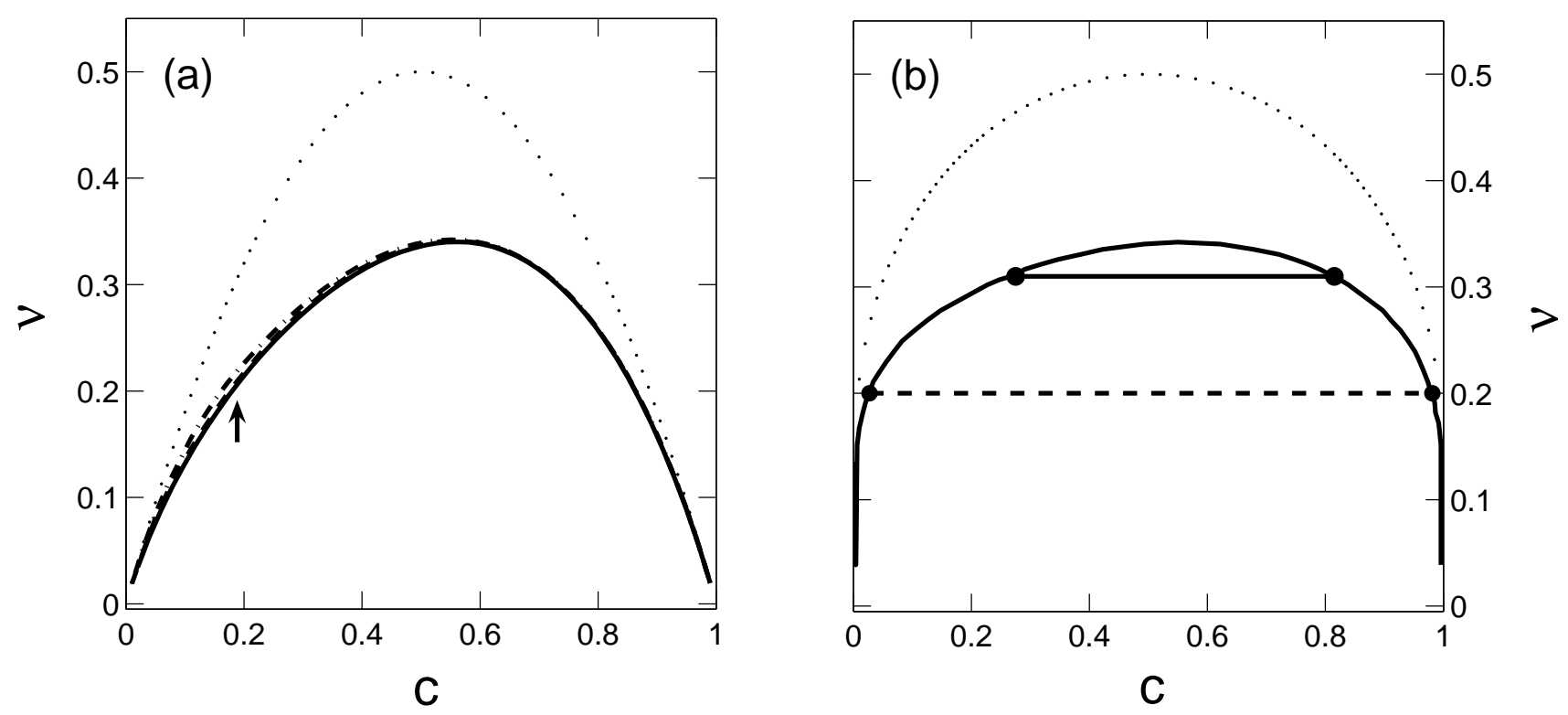

FIG. 3: (a) The spinodal lines of a repulsive surface system are presented as function of the surface coverage $c$. The spinodal is shown for three amounts of added salt. The solid line corresponds to $c_{\mathrm{salt}}=0.1 \mathrm{M}$, the dashed line to $c_{\mathrm{salt}}=0.5 \mathrm{M}$ and the dashed-dotted line to $c_{\mathrm{salt}}=1.0 \mathrm{M}$. All lines share $c^{*}=3, a=5 \AA, v_{\mathrm{ex}}=10 \AA^{3}, \phi_{b}^{2}=10^{-8} \AA^{-3}, Z=1, f=1, b=10 \AA, \nu \varepsilon_{\mathrm{ps}}=0.5 b^{2} a, T=300 \mathrm{~K}, \varepsilon=80$. All three spinodal lines are seen to be almost identical, differing only in the region close to the adsorption-depletion crossover, marked by an arrow. The dotted line marks the pure monolayer spinodal, Eq. (3), and is shown for comparison. (b) The binodal (phase separation) line of a repulsive surface system is presented as function of the surface coverage $c$ for $c_{\text {salt }}=1.0 \mathrm{M}$. Other parameters are the same as in (a). The solid line is the binodal, while the dashed and dashed-dotted lines connect the separated phases for $\nu=0.2$ and $\nu=0.31$, respectively. The dotted line corresponds to the pure-monolayer binodal, Eq. (2), and is shown for comparison.

pure surface one at $c_{c}=0.5$. In Fig. $3 \mathrm{~b}$ we present the binodal line of a repulsive surface for $c_{\text {salt }}=1.0 \mathrm{M}$. The binodal line of the pure surface, Eq. (2), is also presented, and is higher than the numerical binodal for all $c$. Like the spinodal line, the numerical binodal is almost symmetric with respect to the change $c \rightarrow 1-c$. For high $c$ values, all spinodal lines of Fig. 3a overlap almost identically, showing that the dependence of the spinodal on the amount of added salt is very weak. For low $c$ values, careful observation of the three spinodal lines shows a difference between the lines, where the low salt lines are higher than the high salt ones. The three lines join together at $c \sim 0.22$ (see arrow). This difference between the spinodal lines results from the PE depletion at low $c$. For low $c$ values, the spinodal resembles a depletion spinodal, where addition of salt has a strong effect on the spinodal. For higher $c$ values, the surface charge is high enough to allow PE adsorption. All three lines then move toward the electrostatic adsorption spinodal, that is weakly dependent on salt. The point where all lines join together is where the high salt spinodal crosses over from the depletion regime to the electrostatic adsorption regime, and its numerical value agrees with the adsorption-depletion crossover at $1.0 \mathrm{M}$ of salt taken from Fig. 4. The spinodal lines in the different regimes are discussed further in Sec. IV]
In Fig. 4 the adsorbed amount $\Gamma \equiv \phi_{b}^{2} \int_{0}^{\infty} \mathrm{d} x\left(\eta^{2}-1\right)$ is plotted as a function of the surfactant surface coverage $c$ for $c_{\text {salt }}=1.0 \mathrm{M}$. We set $\nu=0.2,0.31$ in Figs. $4 \mathrm{a}, 4 \mathrm{~b}$, respectively. The adsorption is seen to be positive only from a threshold surface coverage $c_{\mathrm{dp}}$, which is similar to the crossover point between spinodal lines shown in Fig. 3. Above this threshold, the adsorbed amount increases linearly with the surface coverage, in agreement with previous results 33, 34]. The threshold is found not to change with $\nu$.

It is interesting to note the effect of the adsorptiondepletion crossover on $\Gamma$ in the coexistence region. In Fig. $3 \mathrm{~b}$ we mark on the binodal line the coexisting phases for $\nu=0.2$ (dashed line) and for $\nu=0.31$ (dashed-dotted line). In Fig. 4 we show $\Gamma$ for the same coexisting phases. Using the lever rule construction [3], $\Gamma$ inside the phase coexistence lies on a straight line between the two marked phases. For $\nu=0.2$ (dashed line in Fig. 4a), the PE adsorbs in one of the phases while depleting from the other. As a result, the adsorption in the phase separated region is always higher than onto a reference single phase (solid line), having the same mean surface coverage $c$, For $\nu=0.31$ the PE adsorbs on the two coexisting phases. The adsorption in the phase separated region (dasheddotted line in Fig. 4b) is almost identical to the adsorption to the single corresponding mixed phase (solid line), 
and there is no gain in the adsorption due to demixing.

The difference between these two behaviors can be qualitatively explained by the following argument. In the electrostatic adsorption case, the adsorbed amount of monomers scales linearly with the surface charge [30], and hence also with $c$. The adsorption of the PE chains aims mainly to balance the surface charge, and since the demixing does not change the overall surface charge, the total adsorbed amount does not change as well. In the case of a depletion phase- adsorption phase coexistence, the charges on the low $c$ (depletion) domain are screened by salt ions, while the higher surface charge domains are screened by the PE chains. In this case, even if the total surface charge is too weak to allow PE adsorption in a single mixed phase, the phase separation allows adsorption at a part of the surface area, and thus allows for a positive adsorbed amount. The crossover between these two behaviors is at $\nu \simeq 0.3$, corresponding to the low $c$ phase being at $c \simeq c_{\mathrm{dp}}$. Namely, that the low $c$ phase is at the adsorption-depletion transition.

Short-Range Attractive Surface The opposite case of a short-range attractive surface is presented in Fig. 5. As can be seen from the figure, the spinodal line is much higher than the corresponding pure-surface spinodal line, and it is far less symmetrical. The addition of salt is shown to increase the spinodal temperature. This increase with salt can be explained by both the screening of the electrostatic repulsion between the lipids, and by the increase in the adsorbed amount of monomers in this case 34], which increases the effective attraction between the lipids. The corresponding adsorbed amount of monomers is shown in Fig. 6. The adsorbed amount here is convex in $c$, showing that the adsorption to a demixed two-phase system is always larger than to a single phase system.

Attractive-Repulsive Crossover In Fig. 7 we show the spinodal lines of monomers for $c^{*}=0.7$ and both $c_{\text {salt }}=1.0 \mathrm{M}$ and $c_{\text {salt }}=0.1 \mathrm{M}$. It is easily seen that the low salt spinodal is higher than the high salt one for $c<c^{*}$ (repulsive surface), while the opposite occurs for $c>c^{*}$, consistent with Fig. 5. Although all three regimes occur in this spinodal, the curve has only one critical point, located near the crossover between the spinodal behavior of the electrostatic adsorption limit to the chemical adsorption limit, at $c \simeq c^{*}$. This shows that the transition of the spinodal line between the regimes is gradual rather than abrupt. The $c_{\text {salt }}=1.0 \mathrm{M}$ line changes convexity at $c \sim 0.2$, creating a "shoulder" in the spinodal. This shoulder marks the transition from a depletion regime to an electrostatic adsorption regime. For extremely strong short-range interactions (very high $\left.\varepsilon_{\mathrm{ps}}\right)$, this shoulder may transform into a triple point on the spinodal. However, we did not find such a triple point numerically for reasonable values of our parameter.

In Fig. 8 the adsorbed amount for $c^{*}=0.7$ is plotted against the surface coverage $c$. For low $c$ values, we see the PEs deplete, similar to Fig. 4, while for higher $c$ the adsorption crosses over from a linear electrostatic adsorp-
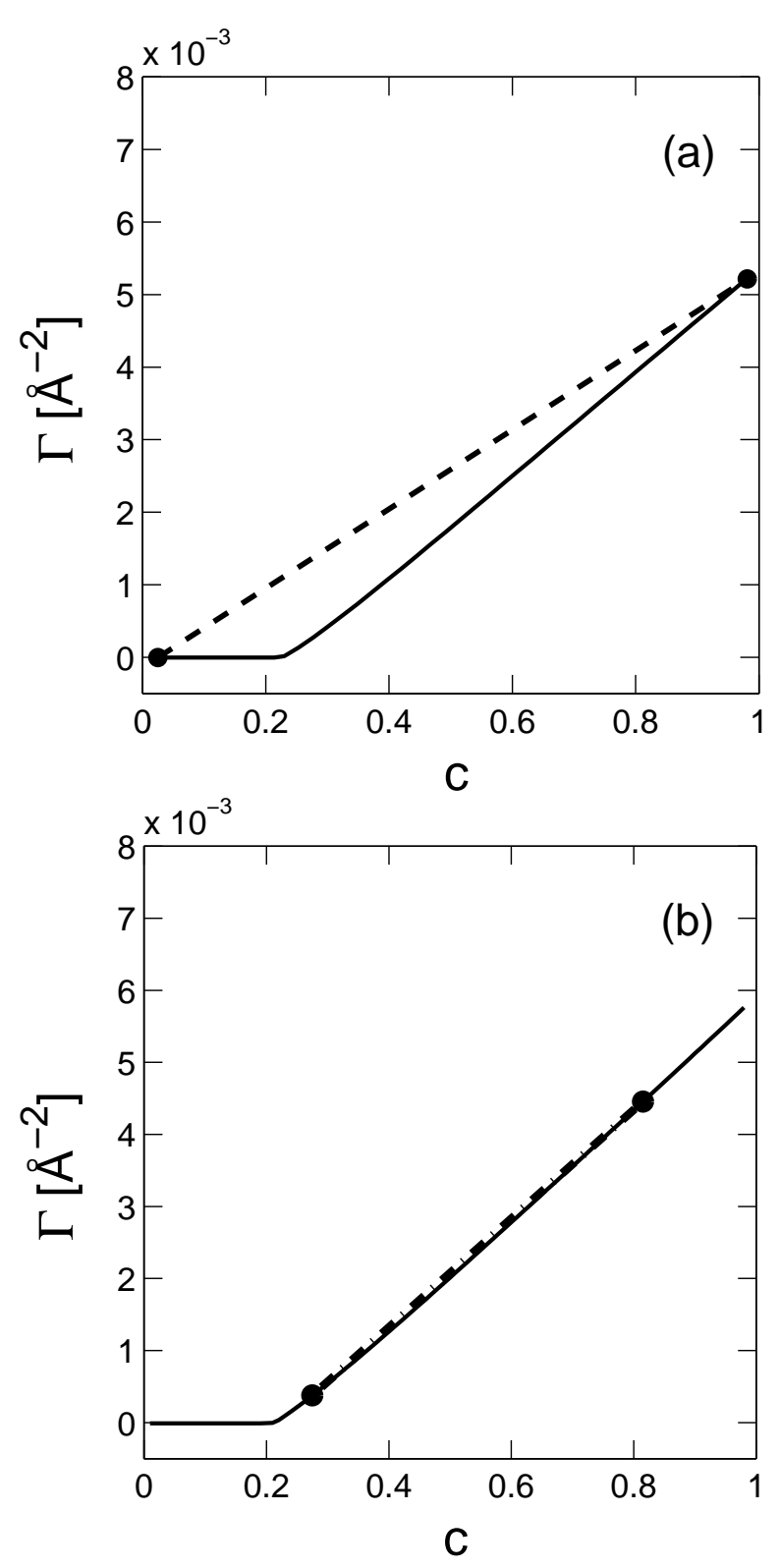

FIG. 4: The adsorbed amount $\Gamma$ is plotted as a function of the surfactant coverage for two values of $\nu$. In (a) the solid line correspond to $\nu=0.2$ while in (b) $\nu=0.31$. Both figures share $c_{\text {salt }}=1.0 \mathrm{M}$, and other parameters are the same as in Fig. 3. In both graphs, a low $c$ depletion regime can be found, in which $\Gamma$ is slightly negative. For larger surface coverage, the adsorbed amount scales linearly with the surface coverage, and no considerable convexity is obtained. The threshold surface coverage for adsorption is unaffected by $\nu$. The dashed line in (a) and dashed-dotted line in (b) are the adsorbed amounts inside the phase coexistence region, and are constructed by the lever rule. A significant increase in adsorption can only be achieved when one of the separated phases is a depletion phase. This is the case in (a). For higher values of $\nu$ (b), the phase separated adsorption line is almost the same as the original (single phase) adsorption line, and no gain in adsorption is achieved. 


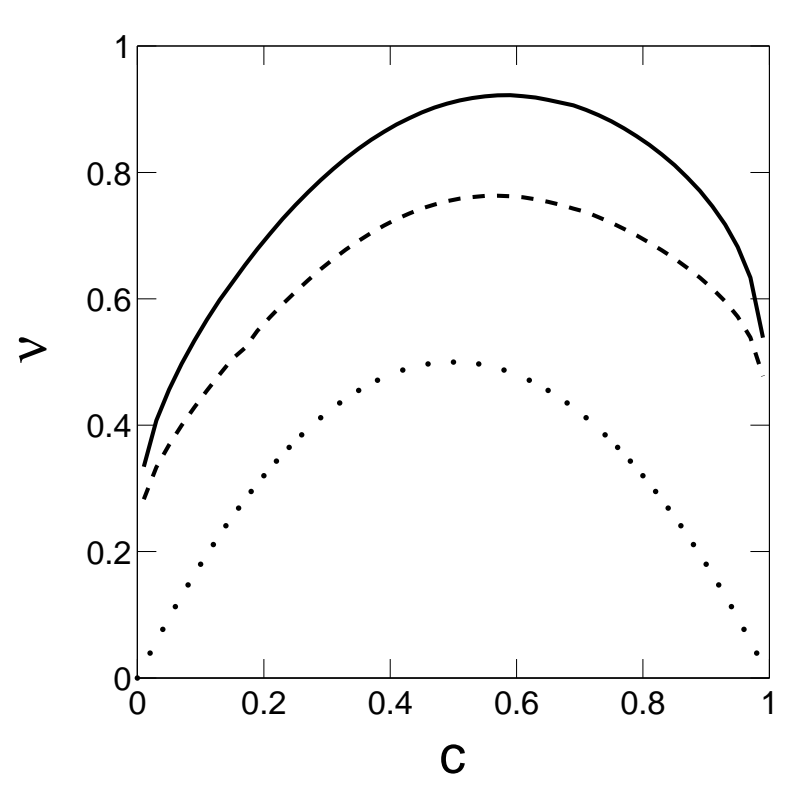

FIG. 5: The spinodal line for a short-range attractive surface, and two salinities: $c_{\text {salt }}=1 \mathrm{M}$ (solid line) and $c_{\text {salt }}=0.1 \mathrm{M}$ (dashed line). The dotted line corresponds to the pure surface spinodal, Eq. (3). The profiles were calculated for $c^{*}=-1$ and $Z=0.1$, and other parameters are the same as in Fig. 3 . In contrast to the repulsive surface case, here the PE adsorption pushes the spinodal to higher temperatures than the spinodal of the pure monolayer. The effect of salt is also more pronounced than in the repulsive surface case, and it is shown that addition of salt increases the spinodal temperature considerably.

tion profile to a convex chemical adsorption profile. The addition of salt decreases the adsorption for low $c$ values, and increases it for higher surface coverage. Note, however, that the crossover between the two salt dependencies is not at $c=c^{*}$ but for a lower $c$. This result is in agreement with a previous one [34], which shows that when the short-range repulsion between the surface and the PE chains becomes small enough, the adsorbed amount starts to increase with $c_{\text {salt }}$ even before the surface becomes attractive, due to an increase in the surface monomer concentration $\eta_{s}^{2}$.

So far we discussed the case where the short-range interactions between the PE chains and the surfactant are more favorable than with the bare surface $\left(\varepsilon_{\mathrm{ps}}>0\right)$. In the case of a neutral polymer, changing the sign of $\varepsilon_{\mathrm{ps}}$ is exactly equivalent to replacing $c^{*} \rightarrow 1-c^{*}$ and $c \rightarrow 1-c$. However, in our case the fact that the surfactant and polymers are oppositely charged breaks this symmetry.

For very strong short-range interactions (large negative $\left.\nu \cdot \varepsilon_{\mathrm{ps}}\right)$ and $0<c^{*}<0.5$, a triple point can emerge in the following way. For low $c$ values, the spinodal resembles the attractive surface spinodal. The spinodal line in this region is always higher than the pure surface spinodal Eq. (3), as was shown in Fig. 5. For $c_{\mathrm{dp}}>c>c^{*}$, the spinodal line decreases substantially to the depletion spinodal, and for $c>c_{\mathrm{dp}}$ it moves to the electrostatic adsorption spinodal. Both of the latter spinodal lines

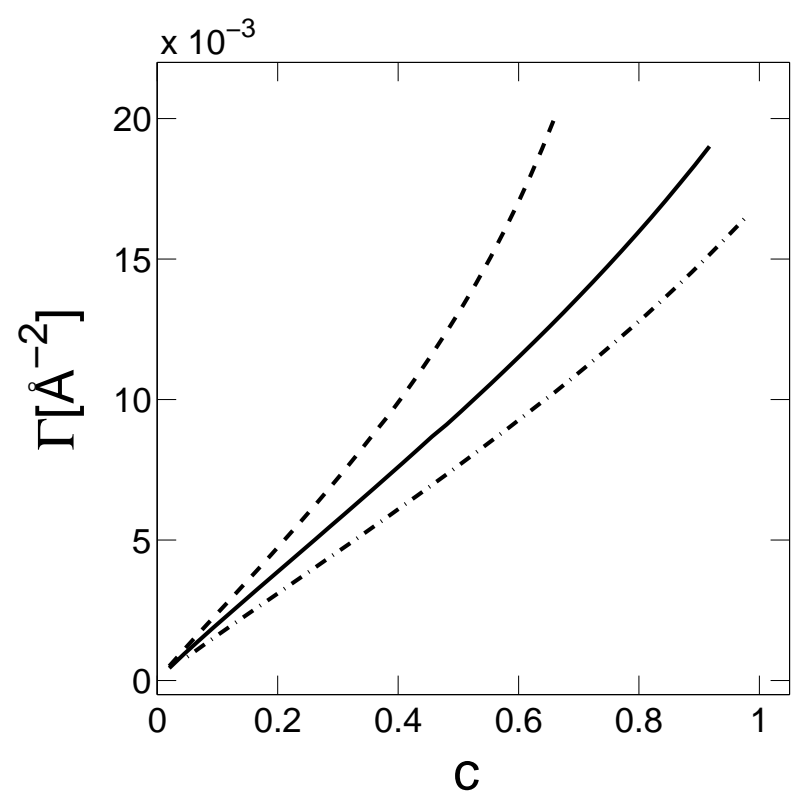

FIG. 6: The adsorbed amounts of monomers are shown for the shortrange attractive surface. The dashed line corresponds to $c_{\text {salt }}=1 \mathrm{M}$, $\nu=0.3$, the solid line to $c_{\text {salt }}=0.5 \mathrm{M}, \nu=0.4$ and the dashed-dotted line to $c_{\text {salt }}=0.5 \mathrm{M}, \nu=0.5$. All profiles share $c^{*}=0$, and other parameters are the same as in Figs. 3. In contrast to the repulsive surface case, here the addition of salt increases the adsorption. An increase in the temperature $\nu$ decreases the adsorption as expected. The adsorption profiles $\Gamma(c)$ are convex with respect to $c$, showing that a demixed lipid monolayer adsorbs more PEs than onto a homogeneous layer with the same mean coverage $c$.

are similar to those in Fig. 3a, both are lower than the pure surface spinodal, and have a critical point at $c_{c} \simeq$ 0.5 . Therefore, there must be a triple point at $c<0.5$, connecting three coexisting phases: (i) a low $c$ chemicaladsorption phase; (ii) a low adsorption phase; and (iii) an electrostatic adsorption phase, as shown in Fig. 9.

In Fig. 9 we show the numerically calculated triple point corresponding to the above conditions. At high temperatures $\nu>0.29$, the phase coexistence for low surface coverage connects a chemical-adsorption phase with either another chemical adsorption phase, or a depletion phase. For higher surface coverage, the phase coexistence connects either a depletion phase or an electrostatic adsorption phase with another electrostatic adsorption phase. A triple point connecting all three phases occurs for $c_{\mathrm{tr}} \simeq 0.22, \nu_{\mathrm{tr}}=0.29$.

\section{ANALYTICAL RESULTS}

In this section we present a theory to explain the three PE-surfactant regimes, and, in particular, aimed at explaining the physics behind the spinodal behavior in each regime. We derive an approximate form for the free energy in each regime, and show the resulting spinodal line. Our model is based on the scaling results of Refs. 30, 33], and is shown to capture the correct behavior of the spin- 


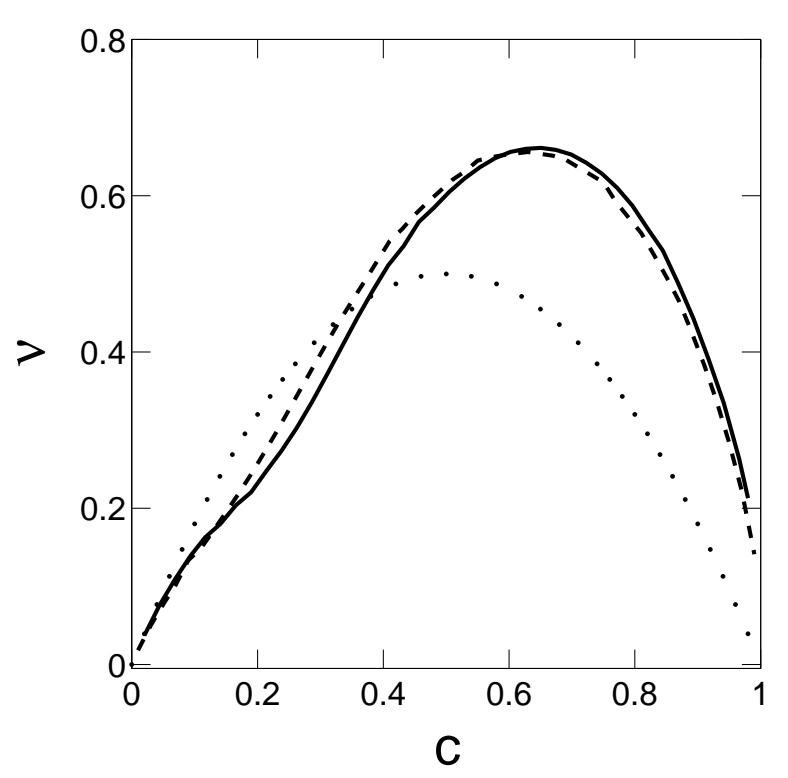

FIG. 7: The spinodal line is presented for $c^{*}=0.7$ for two salinities: $c_{\text {salt }}=0.1 \mathrm{M}$ (dashed line) and $c_{\text {salt }}=1.0 \mathrm{M}$ (solid line). All other parameters are the same as in Fig. 3. The dotted line is the pure surface spinodal, and is presented for comparison. Both spinodals are very close, but one can see that the low salt spinodal line is slightly higher than the high salt spinodal line for $c<c^{*}$, and slightly lower than it for $c>c^{*}$. The saddle point on the high salt spinodal line corresponds to the adsorption-depletion crossover, and occurs at the same surface coverage as in the repulsive surface case. Both spinodals cross over from a low $\nu$ range (lower than the pure surface spinodal) to a high $\nu$ range (resembling an attractive surface) at $c \sim 0.4$.

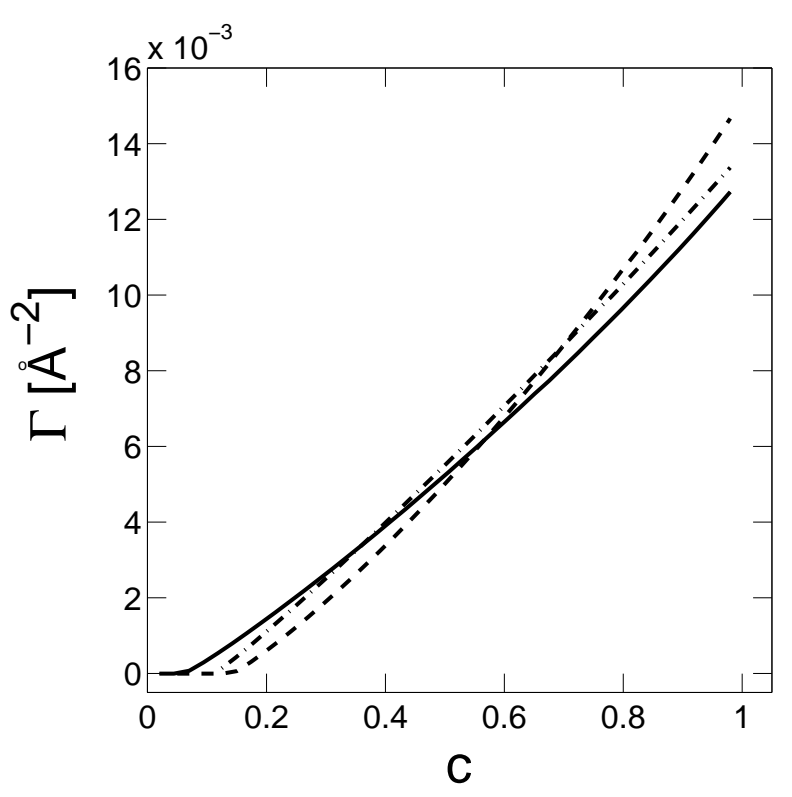

FIG. 8: The adsorbed amount of monomers $\Gamma$ is shown for $c^{*}=0.7$. The solid line corresponds to $c_{\text {salt }}=0.5 \mathrm{M}, \nu=0.3$, the dasheddotted line to $c_{\text {salt }}=1 \mathrm{M}, \nu=0.5$ and the dashed line to $c_{\text {salt }}=1 \mathrm{M}$, $\nu=0.3$. All other parameters are the same as in Fig. 7 . The increase in the amount of salt causes the adsorbed amount to decrease for low $c$, and to increase for high $c$. odal lines in each limit. The analytical equations can be compared to the numerical results with good accuracy, as shown below.

\section{A. Polyelectrolyte Depletion Regime}

When $\varepsilon_{\mathrm{ps}}\left(c-c^{*}\right)<0$ the surface repels the PE chains, and thus the only driving force for adsorption is the electrostatic attraction between the surfactants and the PE chains. This electrostatic attraction strongly depends on the amount of small ions in the solution [33]. When this amount increases beyond a threshold value, the salt ions neutralize the surface and PE charges, and the solution behaves like a neutral polymer solution. In this case, the repulsive short-range interaction between the $\mathrm{PE}$ chains and the surface causes the PE chains to desorb entirely from the surface. This threshold value of salt was previously shown [33] to depend on the surface charge density of the interface and on the charge of the PEs via the scaling law $\left(c_{\text {salt }}^{*}\right)^{3 / 2} \sim|\sigma| f l_{B}^{-1 / 2} a^{-2}$. This relation can be inverted to give a threshold surfactant concentration $c_{\mathrm{dp}}$ :

$$
c_{\mathrm{dp}} \simeq A_{\mathrm{dp}} \frac{c_{\mathrm{salt}}^{3 / 2} l_{B}^{1 / 2} a^{2} b^{2}}{Z f}
$$

below which the PE chains do not adsorb on the charged surface. The prefactor $A_{\mathrm{dp}}$ can be calculated by comparison of Eq. (12) with the numerical results as in Figs. 3-4, yielding $A_{\mathrm{dp}} \simeq 2.35$.

When the PE chains desorb from the surface, the electrostatic potential depends only on the adsorption profile of the small ions. According to the well known PoissonBoltzmann theory [4], this decay is exponential as function of the distance from the surface $x$. This exponential decay causes the interaction term $f \phi_{b}^{2} \eta^{2} \zeta$ between the $\mathrm{PE}$ chains and the electrostatic potential in Eq. (6) to be small in comparison to the other terms. The free energy hence decouples and describes a neutral polymer and an ionic solution. Using the Debye-Hückel approximation for the small electrostatic potentials, the total free energy becomes:

$$
\begin{aligned}
F= & F_{\mathrm{surf}}^{\prime}+\frac{2 \pi l_{B} c^{2} Z^{2}}{\kappa b^{4}}- \\
& b^{2} \frac{a^{2} v_{\mathrm{ex}} \phi_{b}^{4}}{18 \varepsilon_{\mathrm{ps}}\left(c^{*}-c\right)}\left(3+\frac{b^{4} a^{2} v_{\mathrm{ex}} \phi_{b}^{2}}{3 \varepsilon_{\mathrm{ps}}^{2}\left(c^{*}-c\right)^{2}}\right)
\end{aligned}
$$

The second term is the free energy of a solution of small ions under the Debye-Hückel approximation, the third term is the free energy of a neutral polymer solution as in Ref. [3], and the first term $F_{\text {surf }}^{\prime}$ is the free energy of the neutral surfactant monolayer:

$$
\begin{array}{r}
F_{\text {surf }}^{\prime}=b^{-2}[c \ln c+(1-c) \ln (1-c) \\
\left.+\nu^{-1} c(1-c)\right]
\end{array}
$$


where we note that the surface charge term was already taken into account in the small ion solution term of Eq. (13). Differentiating this free energy twice with respect to $c$, we obtain the following equation for the spinodal:

$$
\begin{aligned}
& b^{-2}\left(\frac{1}{c}+\frac{1}{1-c}-2 \nu_{\mathrm{sp}}^{-1}\right)+\frac{4 \pi l_{B} Z^{2}}{\kappa b^{4}} \\
& -b^{2} \frac{a^{2} v_{\mathrm{ex}} \phi_{b}^{4}}{18 \varepsilon_{\mathrm{ps}}\left(c^{*}-c\right)^{3}}\left(6+\frac{4 b^{4} a^{2} v_{\mathrm{ex}} \phi_{b}^{2}}{\varepsilon_{\mathrm{ps}}^{2}\left(c^{*}-c\right)^{2}}\right)=0
\end{aligned}
$$

The first term of the spinodal Eq. (15) is the pure surfactant monolayer spinodal as in Eq. (3), while the second term accounts for the electrostatic interactions between the salt ions and the surface and the third term for the neutral polymer solution [3]. The neutral polymer term, as was shown in Ref. [3], causes the spinodal line to increase slightly. The magnitude of this term is inversely proportional to the short-range interaction strength $\varepsilon_{\mathrm{ps}}$, and can be discarded in the limit of strong short-range repulsion. The salt term, in turn, pushes the spinodal line downwards, as was previously shown by Ref. [4, 5]. The decrease in the spinodal line is substantial, and depends on the amount of added salt. In this (Debye Hückel) approximation, for very large amounts of added salt all electrostatic interactions between the monomers and the surfactants are totally screened, resulting in a strong decrease in $F_{\text {el }}$. The spinodal line then fully recovers the neutral polymer limit from Ref. 3]. For lower amounts of salt, which include most physical cases, the spinodal is pushed downwards from the pure monolayer spinodal, as seen in Fig. 3a.

\section{B. Electrostatic Adsorption Regime}

For $c>c_{\mathrm{dp}}$ and $\varepsilon_{\mathrm{ps}}\left(c-c^{*}\right)<0$, the PEs are attracted to the surface only via their electrostatic interactions, but cannot approach the surface because of the shortrange repulsive interactions. Due to the exclusion from the surface, the PE surface concentration becomes very small, and can be ignored at first approximation.

In previous publications [30, 33, 34], we discussed at length the adsorption of polyelectrolytes to charged surfaces, which have short-range repulsive interactions with the PE chains. We summarize here the main results. It was shown that the dominant interaction is the electrostatic interaction between the PE and the surface. The excluded volume interaction was found to be negligible in the numerical solutions of the mean-field Eqs. (89). The $\mathrm{PE}$ adsorption was shown to decrease with the addition of salt 33, 34 for low amounts of added salt, while for higher amounts the polymer chains were shown to desorb entirely from the surface [33]. It was shown that the adsorbed polyelectrolyte layer can be characterized by a single (rescaled) monomer concentration scale (denoted by $\eta_{M}^{2}$ ) and a single length scale for the adsorbed layer

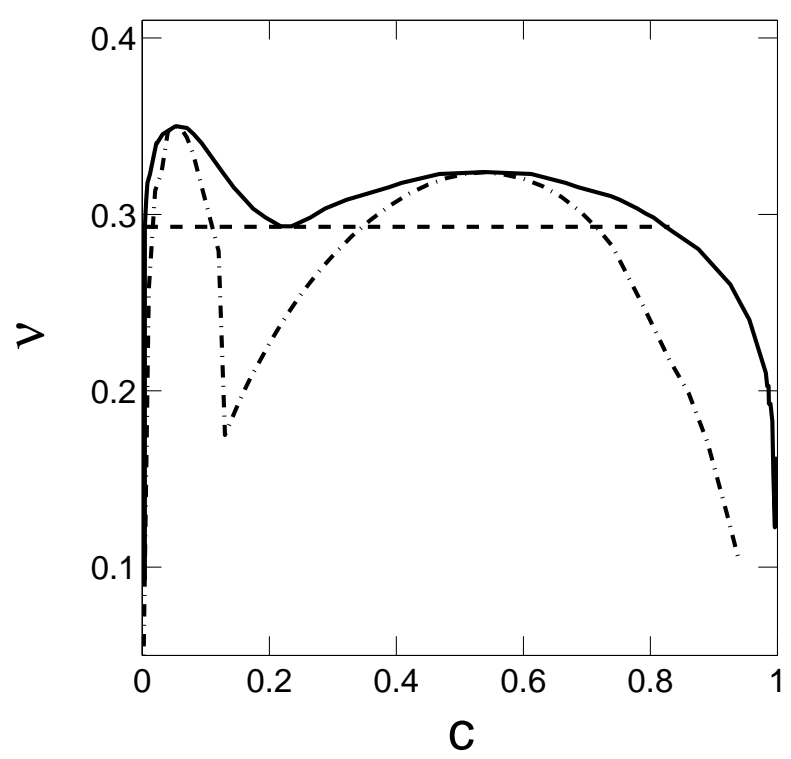

FIG. 9: Binodal and spinodal lines exhibiting a triple point. The dashed-dotted line is a numerically calculated spinodal line, for the following parameters: $a=5 \AA, b=10 \AA ̊, \nu \cdot \varepsilon_{\mathrm{ps}}=-1.0 b^{2} a, f=0.3$, $Z=1, c^{*}=0.1 c_{\text {salt }}=1 \mathrm{M}, T=300 \mathrm{~K}, \phi_{b}^{2}=10^{-8} \AA^{-3}, v_{\text {ex }}=$ $10 \AA^{3}$. The solid line represents the corresponding binodal line. $A$ triple point, connecting three coexisting phases, exists for $\nu_{\mathrm{tr}} \simeq 0.29$. The three coexisting phases are connected by the dashed line.

width (denoted by $D$ ). The free energy of the solution can be calculated using a Flory-like approximation [30]. While the full details can be found in the previous publications, we present here the main results:

$$
\begin{array}{r}
\frac{F_{\text {ads }}}{k_{B} T}=A_{1} \frac{a^{2}}{6} \frac{\phi_{b}^{2} \eta_{M}^{2}}{D}-A_{2} f|\sigma| l_{B} D^{2} \phi_{b}^{2} \eta_{M}^{2}+ \\
A_{3} f^{2} l_{B} \phi_{b}^{4} \eta_{M}^{4} D^{3}+A_{4}|\sigma|^{2} l_{B} D
\end{array}
$$

where the surface charge is denoted by $\sigma=-c Z / b^{2}$. The first term is the elasticity term of the polyelectrolyte, the second is the electrostatic attraction between the surface and the monomers, and the third is the electrostatic repulsion between monomers. This repulsion is assumed to be strong enough to dominate over the excluded volume interactions. The last term is the interaction of the electrostatic potential and the surface charge, where the rescaled potential is $\left|\zeta_{s}\right|=A_{4}|\sigma| l_{B} D$. The short-range interaction between the surfactants and the PE chains depends on the surface monomer concentration, and can be ignored because of the strong short-range repulsion. Minimization of Eq. (16) with respect to $D$ and $\eta_{M}^{2}$ yields: 


$$
\begin{aligned}
D & \simeq\left(\frac{a^{2}}{l_{B} f \sigma}\right)^{1 / 3} \\
\phi_{b}^{2} \eta_{M}^{2} & \simeq\left(\frac{\sigma^{2} \sqrt{l_{B}}}{a f}\right)^{2 / 3} \\
F_{\text {ads }} & \simeq I_{0}\left(\frac{a^{2} l_{B}^{2}}{f}\right)^{1 / 3}|\sigma|^{5 / 3}
\end{aligned}
$$

where the prefactor $I_{0}$ can be shown to be positive. In this case, the full free energy can be written as:

$$
\begin{aligned}
F & =I_{0}\left(\frac{a^{2} l_{B}^{2} Z^{5}}{f b^{10}}\right)^{1 / 3} c^{5 / 3} \\
& +b^{-2}\left[c \ln c+(1-c) \ln (1-c)+\nu^{-1} c(1-c)\right] .
\end{aligned}
$$

Note that this free energy has a fractional power (nonanalytic) in $c$. The spinodal equation is obtained by differentiating Eq. (20) twice with respect to $c$, yielding:

$$
2 \nu_{\mathrm{sp}}^{-1}=\frac{1}{c}+\frac{1}{1-c}+\frac{10}{9} I_{0}\left(\frac{a^{2} l_{B}^{2} Z^{5}}{f b^{4}}\right)^{1 / 3} c^{-1 / 3}
$$

Eq. (21) shows that the spinodal temperature $\nu_{\mathrm{sp}}$ is always lower than that of the pure surfactant monolayer, Eq. (31). This result is consistent with results derived for the spinodals of protein solutions $[4,6]$. The critical point is the maximum of this spinodal line $\nu_{\mathrm{sp}}(c)$ in Eq. (21), satisfying:

$$
\frac{2 c_{c}-1}{c_{c}^{2}\left(1-c_{c}\right)^{2}}=\frac{10}{27} I_{0}\left(\frac{a^{2} l_{B}^{2} Z^{5}}{f b^{4}}\right)^{1 / 3} c_{c}^{-4 / 3} .
$$

The critical point is at $c>0.5$, regardless of the sign of $\varepsilon_{\mathrm{ps}}$. For our chosen parameters of $a=5 \AA, I_{0}=2.8$, $l_{B} \simeq 7 \AA, b=10 \AA$ and $Z=1$ the critical concentration from Eq. (22) turns out to be at $c=0.5365$, showing that the spinodal is almost symmetric, in agreement with Fig. 3.

\section{Strong Short-Range Attraction Regime}

For large enough $\varepsilon_{\mathrm{ps}}\left(c-c^{*}\right)>0$ (strong short range attraction) the adsorbing polymer layer is highly charged, more than the initial surface [34, 35]. In this case, the main electrostatic contribution to the free energy comes from the monomer-monomer repulsion, rather than the electrostatic attraction to the surface.

When the amount of small ions in the solution is small, the length scale for electrostatic potential decay is the same as that of the monomer concentration. The single length scale allows us to use similar scaling ideas as in the repulsive surface case. The highest monomer concentration occurs at the surface itself $\eta_{\max }=\eta_{s}$, and acts as the scale for the monomer concentration. Similarly to Ref. [30], we can produce scaling rules for the monomer adsorption using a Flory-like free energy. The free energy in this case is:

$$
\begin{array}{r}
\frac{F_{\text {ads }}}{k_{B} T}=A_{1} \frac{a^{2}}{6} \frac{\phi_{b}^{2} \eta_{s}^{2}}{D}-\frac{1}{2} b^{-2} \varepsilon_{\mathrm{ps}}\left(c-c^{*}\right) \phi_{b}^{2} \eta_{s}^{2} \\
+A_{3} f^{2} l_{B} \phi_{b}^{4} \eta_{s}^{4} D^{3}
\end{array}
$$

where the first term is again the contribution of the chain elasticity, the second term is the short-range attraction of the polymer chains to the surface, and the last term is the electrostatic repulsion between the monomer layers. The electrostatic interactions between the surface and the monomer concentrations are assumed to be small in comparison to the short-range ones, and are omitted from this free energy. Minimization of this free energy yields the following scales for $D$ and $\eta_{s}^{2}$ :

$$
\begin{aligned}
D & \simeq \frac{a^{2} b^{2}}{\varepsilon_{\mathrm{ps}}\left(c-c^{*}\right)} \\
\eta_{s}^{2} & \simeq \frac{\varepsilon_{\mathrm{ps}}^{4}\left(c-c^{*}\right)^{4}}{b^{8} a^{6} l_{B} f^{2} \phi_{b}^{2}} \\
\frac{F_{\mathrm{ads}}}{k_{B} T} & \simeq-I_{1} \frac{\varepsilon_{\mathrm{ps}}^{5}\left(c-c^{*}\right)^{5}}{b^{10} a^{6} l_{B} f^{2}}
\end{aligned}
$$

where $I_{1}$ is a constant of order unity. The adsorption free energy is negative, as expected, and is very strongly dependent on the strength of the short range interactions. The total free energy is now the sum of the pure monolayer free energy and the adsorption free energy, and the spinodal line can be readily obtained as:

$$
\frac{1}{c(1-c)}-2 \nu_{\mathrm{sp}}^{-1}-20 I_{1} \frac{\Theta^{5}\left(c-c^{*}\right)^{3}}{\nu_{\mathrm{sp}}^{5} l_{B} a^{6} b^{8} f^{2}}=0
$$

where we denote $\Theta \equiv \nu \cdot \varepsilon_{\text {ps }}$. In this case, the spinodal temperature $\nu_{\mathrm{sp}}$ is always larger than the corresponding temperature of the pure-surface spinodal. The critical point is obtained by differentiating Eq. (27) once more with respect to $c$.

$$
-\frac{1}{c_{c}^{2}}+\frac{1}{\left(1-c_{c}\right)^{2}}-60 I_{1} \frac{\Theta^{5}\left(c_{c}-c^{*}\right)^{2}}{\nu_{c}^{5} l_{B} a^{6} b^{8} f^{2}}=0
$$

which for $\Theta>0$ gives $c_{c}>0.5$, and vice versa. The resulting spinodal line is less symmetric than in the 

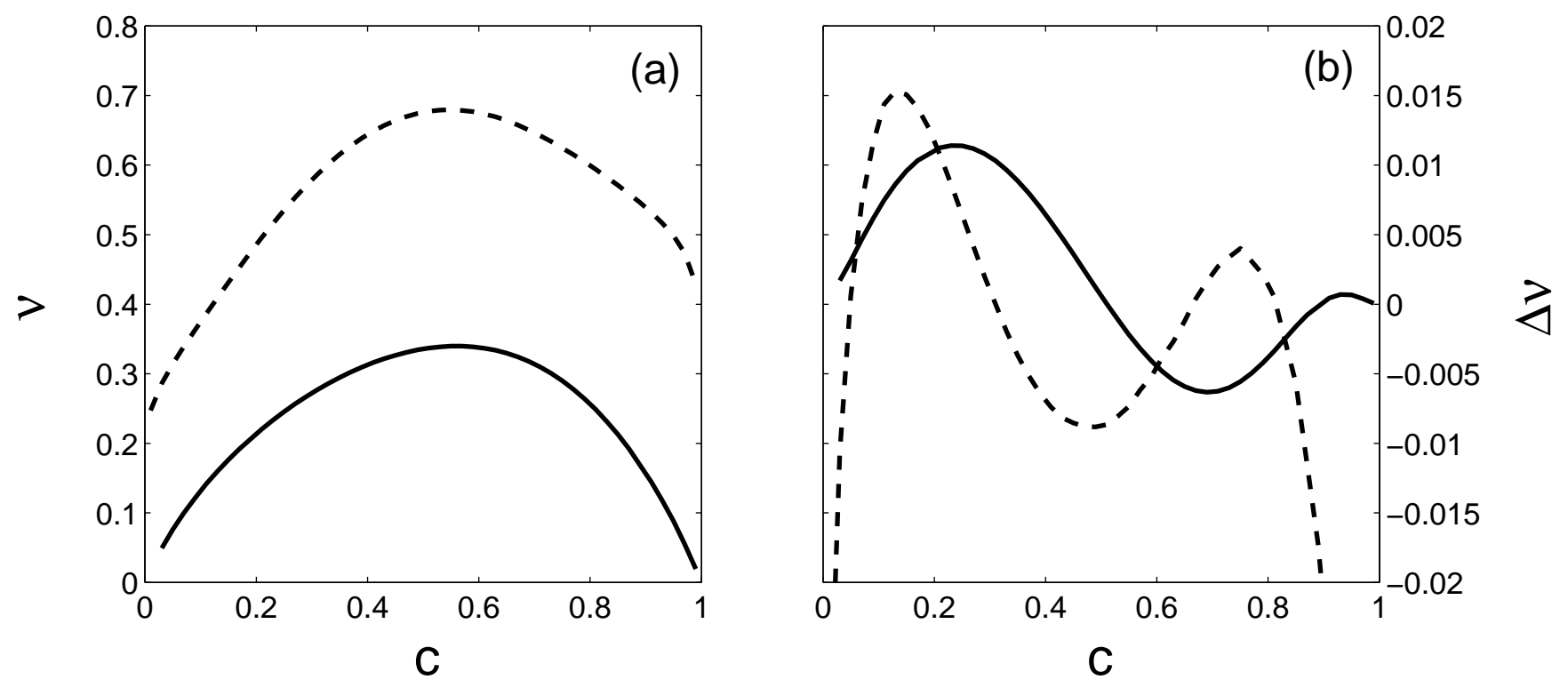

FIG. 10: Comparison between the numerical and analytical spinodal lines. In (a) we present the numerical spinodal lines for two sets of parameters. The solid line is the numerical spinodal line in the repulsive case for $Z=1, c^{*}=3.0$, while the dashed line is the numerical spinodal line in the attractive case, for $Z=0.1, c^{*}=-1.0$. Both spinodal lines share $a=5 \AA, b=10 \AA, \nu \cdot \varepsilon_{\mathrm{ps}}=0.5 b^{2} a, f=1, c_{\mathrm{salt}}=0.01 \mathrm{M}$, $T=300 \mathrm{~K}, \phi_{b}^{2}=10^{-8} \AA^{-3}, v_{\mathrm{ex}}=10 \AA^{3}$. We do not show the analytical spinodals here since they are too close to the numerical ones to be drawn. In (b) we present the differences between the analytical calculations and the numerical spinodals. The solid and dashed lines are the difference between the spinodal lines in Eqs. (21) and (27), respectively, with $I_{0}=2.8$ and $I_{1}=0.023$, and the numerical spinodals in (a). The differences are small, and the numerical spinodal lines are well characterized by the analytical approximation.

electrostatic adsorption case, and the critical point is further from the pure surface value of 0.5 than the repulsivesurface spinodal Eq (21). This result is also in agreement with the numerical results of Fig. 5.

\section{Comparison of the analytical and numerical results}

Both spinodals, Eqs. (2127), contain one free parameter each, and can be compared to the numerical spinodals. The comparison is presented in Fig. 10. As can be seen, both of the spinodal equations show good agreement with the numerical lines, using the values $I_{0}=2.8$ for the repulsive surface case and $I_{1}=0.023$ for the attractive surface case.

The analytical model given above for the two adsorption regimes is accurate only for the case of low amounts of added salt. This is because the main assumption is the existence of a single length scale for the decay of the electrostatic potential and the monomer concentration. More work is necessary to extend the present model for the high salt regime.

\section{CONCLUSIONS}

We present numerical and analytical results for the phase separation of a surfactant monolayer in presence of polyelectrolytes (PEs). We show that the short-range interactions between the surfactant and polyelectrolytes have a strong impact on the surfactant critical temperature and critical concentration. We also present analytical results in two limits: (i) strong short-range repulsive, and (ii) attractive surfactant-polyelectrolyte interactions.

For short-range repulsive interactions, our results show that surfactant phase separation occurs for lower temperatures than for a non-interacting surfactant layer. For such systems, the polyelectrolyte chains may desorb completely from the low surfactant concentration domains, while for higher concentration phases the PE adsorption increase linearly with the surfactant surface coverage. The critical surfactant concentration slightly deviates from the bare one $c_{c}=0.5$, showing nearly symmetrical spinodal and binodal lines. In addition, we find a temperature range in which phase separation does not increase the PE adsorption. For lower temperatures, where the phase separation occurs between a strongly adsorbing phase and a depleting phase, the gain in adsorption becomes substantial. For short-range attractive interactions, in contrast, the phase separation al- 
ways increases adsorption, and the demixing temperatures are always larger than the pure-surface ones. The critical point moves more significantly away from the pure-surface symmetric value. The convexity of the adsorption is more pronounced, showing that phase separation indeed increases the PE adsorption on the surfactant monolayer. In intermediate cases, where the short-range interaction between the polyelectrolyte chains and the surface changes sign as function of the surfactant surface coverages, a triple point can be found on the binodal line connecting three coexisting phases on the same surface.

The addition of salt is shown to increase the threshold surfactant-concentration for the desorption phase. It has a weaker effect on the spinodal line for the case of electrostatic adsorption. For short-range adsorbing surfaces, the addition of salt increases the spinodal line considerably.

Our results are derived within mean-field, using the ground state dominance approximation. This treatment neglects correlation effects between the different constituents, and they may be strong in some cases. We also assume that the lipids are entirely insoluble, and neglect the effects of the line tension between the surfactant phases. Despite these drawbacks, we believe that our main results still hold. Our results may serve as a starting point for more complex models.

Acknowledgments: We thank Avinoam Ben-Shaul, Yoram Burak and Shelly Tzlil for helpful discussions. Support from the Israel Science Foundation (ISF) under grant no. 160/05 and the US-Israel Binational Foundation (BSF) under grant no. 287/02 is gratefully acknowledged.
[1] P.G. de Gennes, J. Phys. Chem. 94, 8407 (1990).

[2] D. Andelman, J-F Joanny, J. Phys II (France) 3, 121 (1993).

[3] X. Chatellier, D. Andelman, J. Phys. Chem. 100, 9444 (1996).

[4] D. Harries, S. May, A. Ben-Shaul, Coll. and Surf. A 208, 41 (2002).

[5] S. May, D. Harries, A. Ben-Shaul, Phys. Rev. Lett. 89, 268102 (2002).

[6] C. L. Baciu, S. May, J. Phys.: Condens. Matter 16, S2455 (2004).

[7] E.C. Mbamala, A. Ben-Shaul, S. May, Biophys. J. 88, $1702(2005)$.

[8] S. Tzlil, M. Deserno, W.M. Gelbart, A. Ben-Shaul, Biophys. J. 86, 2037 (2004).

[9] S. Tzlil, A. Ben-Shaul, BioPhys. J. 89, 2972 (2005).

[10] D. von Effenterre, D. Roux, Europhys. Lett. 64, 543 (2003).

[11] O. González-Amezcua, M. Hernández-Contreras, J. Chem. Phys. 121, 10742 (2004).

[12] May S., J. Phys:Condens. Matter 17, R833 (2005).

[13] P.M. Biesheuvel, M. van der Veen, W. Norde, J. Phys. Chem. B 109, 4172 (2005).

[14] C. Fleck, R.R. Netz, H.H. von Grünberg, Biophys. J. 82, 78 (2002).

[15] Z. Leonenko, D. Cramb, Nano Letters 2, 305 (2002).

[16] Z. Leonenko, D. Merkle, S.P. Lees-Miller, D.T. Cramb, Langmuir 18, 4873 (2002).

[17] Z.V. Leonenko, E. Finot, H. Ma, T.E.S. Dahms, Cramb D.T, Biophys. J. 86, 3783 (2004).

[18] B. Maier, J.O. Rädler, Macromolecules 33, 7185 (2000).

[19] C. M. Wu, L. Willisa, H.L. Chen, T.L. Lin, U.S. Jeng, Macromolecules 37, 4974 (2004).

[20] A. Ianoul, P. Burgos, Z. Lu, R.S. Taylor, L.J. Johnston, Langmuir 19, 9246 (2003).

[21] D. Merkle, S.P. Lees-Miller, D.T. Cramb, Biochemistry 43, 7263 (2004).
[22] C. Monteux, M-F Llauro, D. Baigl, C.E. Williams, O. Anthony, V. Bergeron, Langmuir 20, 5358 (2004).

[23] C. Monteeux, C.E. Williams, V. Bergeron, Langmuir 20, 5367 (2004).

[24] F.W. Wiegel, J. Phys A: Math. Gen. 10, 299 (1977).

[25] M. Muthukumar, J. Chem. Phys. 86, 7230 (1987).

[26] X. Chatellier and J.F. Joanny, J. Phys. II (France) 6, 1669 (1996).

[27] R. Varoqui, A. Johner, A. Elaissari, J. Chem. Phys. 94, 6873 (1991).

[28] R. Varoqui, J. Phys. II (France) 3, 1097 (1993).

[29] J.F. Joanny, Eur. Phys. J. B. 9, 117 (1999).

[30] I. Borukhov, D. Andelman, H. Orland, Macromolecules 31, 1665 (1998); Europhys. Lett. 32, 499 (1995).

[31] I. Borukhov, D. Andelman, H. Orland, Eur. Phys. J. B 5, 869 (1998).

[32] I. Borukhov, D. Andelman, H. Orland, J. Phys. Chem. B 103, 5042 (1999).

[33] A. Shafir, D. Andelman, R.R. Netz, J. Chem. Phys. 119, 2355 (2003).

[34] A. Shafir, D. Andelman, Phys. Rev. E 70, 061804 (2004).

[35] Q. Wang, Macromolecules 38, 8911 (2005).

[36] O.V. Borisov, E.B. Zhulina, T.M. Birshtein, J. Phys. II (France) 4, 913 (1994).

[37] A.V. Dobrynin, A. Deshkovski, M. Rubinstein, Macromolecules 34, 3421 (2001).

[38] P.G. de Gennes, Macromolecules 14, 1637 (1981).

[39] W.H. Press, B.P. Flannery, S.A. Teukolsky, W.T. Vetterling, Numerical Recipes in C: The Art of Scientific Computing, (Cambridge University, Cambridge 1992), Chap. 17, p. 762.

[40] D. Andelman, in "Handbook of Biological Physics: Structure and Dynamics of Membranes", Vol. 1B, edited by R. Lipowsky and E. Sackmann, (Elsevier Science B.V., Amsterdam, 1995), Chap. 12, p. 603. 